\title{
Qualitätssicherung operative Gynäkologie - Einführung
}

Die Entwicklung und Anpassung einer Gebührenordnung ist ein wichtiger Prozess in einem freien Gesundheitswesen, das die Interessen der Patienten ebenso zu beachten hat wie die Bedürfnisse der so genannten Leistungserbringer in Klinik und Praxis. Die Erfahrungen in Ländern mit rein staatlicher Lenkung und ohne Rücksicht auf wirtschaftliche Gegebenheiten und Notwendigkeiten - das gilt nicht nur für den ehemaligen Ostblock - zeigen übereinstimmend ein Absinken der Behandlungs- und Ergebnisqualität zum Schaden der Patienten, sowie die Entwicklung einer Zwei-Klassen-Medizin, die wir in Deutschland bisher so gut wie nicht kannten. Dabei dürfen wir allerdings nicht übersehen, dass wir in Deutschland bisher auf einer Insel der Glückseligkeit lebten, wenn man sich überlegt, wo in unserer Nachbarschaft diese Zwei-Klassen-Medizin unbekannt war. Mir fallen da keine östlichen und nur sehr wenige westliche Länder ein.

Die unübersehbaren Versuche der Politik (und hier nicht nur der jetzigen Regierung) nach Budget, staatlicher Reglementierung, Kontrolle, Dominanz der Kostenträger etc. sind freiheitsfremde Instrumente, die unser deutsches Gesundheitssystem dem des früheren Ostens anzugleichen drohen. Hier wird es nur Verlierer geben. Es ist zu hoffen, aber nicht zuverlässig zu erwarten, dass uns in Deutschland dieser Weg zur Staatsmedizin erspart bleibt.

Entgeltsysteme haben in mancherlei Hinsicht ausgeprägte Gemeinsamkeiten mit Qualitätssicherungsmaßnahmen. Zum einen versuchen sie stets die Pauschalierung: eine abzurechnende Leistung wird standardisiert, damit sie unabhängig von verschiedenen variablen Parametern wie Zeitdauer, Schwierigkeitsgrad, persönlichem Geschick und Erfahrung Allgemeingültigkeit erlangt. Zum anderen beschreibt die Vielzahl der Leistungen in einem Entgeltsystem den derzeitigen medizinischen Stand diagnostischer und therapeutischer Verfahren.

Auch die Auswirkungen eines Entgeltsystems auf die Anwendung dieser verschiedenen diagnostischen und therapeutischen Verfahren, sowie auf die Qualität ärztlicher Berufsausübung sind beachtlich: Da Kliniken und Arztpraxen aus wirtschaftlicher Sicht Unternehmen sind, die sich selbst tragen und auch einen Gewinn zur Selbsterhaltung und Weiterentwicklung erzielen müssen, werden unterbezahlte Leistungen zurückgehen und höher bewertete Leistungen zunehmen. Hier sind gefährliche Eingriffe in den Versorgungsbedarf und in das praktische Procedere von Diagnostik und Therapie denkbar. Zur Weiterentwicklung der GOÄ und zur Anpassung an moderne medizinwissenschaftliche Entwicklungen gehört daher ein erhebliches Augenmaß.

Es war daher notwendig, dass sich die Deutsche Gesellschaft für Gynäkologie und Geburtshilfe als wissenschaftliche Fachgesellschaft gemeinsam mit dem Berufsverband der Frauenärzte Gedanken um diese Verknüpfungen zwischen Qualität und Entgelt machte und eine gemeinsame Kommission ins Leben rief, die die GOÄ zu aktualisieren hatte. Das Ergebnis liegt hier vor und kommentiert die Gebührenordnung vor allem aus der Sicht der Qualitätssicherung. Den Autoren war es ein Anliegen, diese genannte Verknüpfung sichtbar zu machen - es ist ihnen gelungen und es ist ihnen zu danken. 